\title{
From Two-Dimensional Metal-Organic Coordination Networks to Near-Infrared Luminescent PbS Nanoparticle/Layered Polymer Composite Materials
}

\author{
Fang Cui ${ }^{1}$, Junhu Zhang ${ }^{1}$, Tieyu Cui ${ }^{2}$, Sen Liang ${ }^{1}$, Bao Li ${ }^{1}$, Quan Lin ${ }^{1}$, and Bai Yang ${ }^{1}(\bowtie)$ \\ ${ }^{1}$ State Key Lab of Supramolecular Structure and Materials, College of Chemistry, Jilin University, Changchun 130012, China \\ ${ }^{2}$ Shenyang National Laboratory for Materials Science, Institute of Metal Research Chinese Academy of Sciences, Shenyang 110016 , \\ China
}

Received: 10 April 2008/ Revised: 6 June 2008/Accepted: 24 June 2008

CTsinghua Press and Springer-Verlag 2008. This article is published with open access at Springerlink.com

\begin{abstract}
Two-dimensional (2-D) layered metal-organic coordination (lead methacrylate (LDMA)) networks have been prepared in aqueous solution under mild conditions and their structure determined by single crystal diffraction. As the ligand used in our experiments is easily polymerized, these metal-organic coordination layers are therefore employed as precursors to fabricate cross-linked polymer layered materials through $\gamma$-irradiated polymerization. The stabilities of the samples are significantly improved after $\gamma$-irradiation. To our knowledge, this is the first time that covalent bonded polymer layered structures have been fabricated without the assistance of added surfactant or template. Such layered polymer materials cannot only act as alternatives to layered inorganic materials in some caustic environments, but also allow the generation of PbS nanoparticles (NPs) without aggregation in the polymer matrix. By exposing the polymer layer to $\mathrm{H}_{2} \mathrm{~S}$ gas at room temperature, uniform PbS nanoparticles with an average size of about $6 \mathrm{~nm}$ are generated in situ. In addition, the resulting PbS NPs exhibit near-infrared (NIR) luminescent properties, which suggests the composite materials may be useful as active optical elements at communication wavelengths from 1300 to $1550 \mathrm{~nm}$.
\end{abstract}

\section{KEYWORDS}

$\mathrm{PbS}$ nanoparticles, layered material, near-infrared, $\gamma$-irradiated polymerization, in situ

\section{Introduction}

The functionalization of polymer materials by the incorporation of semiconductor nanoparticles (NPs) is of great importance in current chemistry, physics, and materials science because of the potential applications of the resulting materials in fabricating devices with optical, electrical, and magnetic properties [1]. Bulk PbS is a direct-bandgap IV-VI semiconductor with a small bandgap of $0.41 \mathrm{eV}$ and an exciton Bohr radius of $18 \mathrm{~nm}$ at room temperature, which means that an obvious quantum confinement effect can be observed in PbS NPs [2]. A number of studies related to the synthesis and characterization of nanocrystalline $\mathrm{PbS}$ have been reported in Refs. $[3,4]$. From a technological perspective, PbS NPs are of interest because their third-order nonlinear optical response is expected to be huge (30 times

Address correspondence to byangchem@jlu.edu.cn

\section{算 Springer}


that of GaAs and 1000 times that of CdSe) [5, 6]; they are thus useful materials for optical devices such as optical switches. Also, $\mathrm{PbS}$ is among the few materials that can provide size-tunable optical transitions at important near-infrared (NIR) wavelengths $[7,8]$. Diverse applications, such as integrated optoelectronic systems operating at wavelengths of $1.3-1.55 \mu \mathrm{m}$ that are important to telecommunication $[9,10]$, microscopic imaging in the "biological transparency window" [11], photodetectors [12], highly efficient solar cells [13], and tunable NIR lasers [14] may be enabled or vastly improved through the incorporation of PbS NPs. PbS NPs/ polymer nanocomposites in the forms of bulk optical materials [15], thin-film materials [16], and even one-dimensional (1-D) fibriform materials [17] have been extensively studied; however, the preparation of well-dispersed PbS NPs incorporated in layered polymer matrices has never been reported.

Increasing attention has been paid to twodimensional (2-D) layered materials because they are promising materials for adsorption, ion exchange and storage, catalyst carriers, intercalation reactions, and immobilization of biological materials [18-22]. Layered inorganic materials, such as clay minerals, phosphates, layered double hydroxides (LDHs), and silicates, have been intensively studied. Recently, 2-D layered metal-organic coordination networks have received steadily growing interest due to their enormous variety of interesting molecular topologies and wide potential as functional solid materials [23-29]. Although numerous metalorganic coordination networks possessing claylike structures have been successfully prepared, it is still a great challenge to synthesize novel layered materials with different components as well as to further explore their properties and applications. Herein, we report the preparation of 2-D lead methacrylate (LDMA) networks, and that these metal-organic coordination networks can be employed as precursors to fabricate $\mathrm{PbS}$ NPs/layered polymer composite materials through the combined use of $y$-irradiated polymerization and a gas/solid reaction. To our knowledge, this is the first time that covalently bonded polymer layered structures have been fabricated without the assistance of added surfactant or template. Such layered polymer materials cannot only act as alternatives to layered inorganic materials in some caustic environments, but also favor formation of uniform PbS NPs throughout the entire polymer matrix. In addition, the resulting PbS NPs exhibit NIR luminescent properties, allowing the composite materials to be useful as active optical elements at communication wavelengths.

\section{Experimental}

\subsection{Chemicals and materials}

$\mathrm{PbO}$ and methacrylic acid (MA) were purchased from Beijing Chemical Reagent Company (Beijing, China). All chemicals were of analytical reagent grade and used as received. The water used in the experiments was deionized with a resistivity of $18 \mathrm{M} \Omega / \mathrm{cm}$.

\subsection{Synthesis of LDMA layered materials}

In a typical synthesis, $\mathrm{PbO}(28 \mathrm{~g})$ was added to a 500 $\mathrm{mL}$ three-necked flask containing MA $(22 \mathrm{~mL})$ and distilled water $(125 \mathrm{~mL})$. The reaction mixture was stirred continuously for at least $4 \mathrm{~h}$ at $60^{\circ} \mathrm{C}$ to ensure the complete dissolution of $\mathrm{PbO}$. After hot filtration, ethanol was added to the filtrate, and the solution was kept at $20{ }^{\circ} \mathrm{C}$ for several weeks. White flaky crystals (length and width: from several millimeters to more than one centimeter; thickness: from several tens to several hundred micrometers) slowly separated out, and were collected by filtration and then washed with a small quantity of water. Finally, the resulting white crystals were dried in air at room temperature.

\subsection{Preparation of poly(lead methacrylate) (P-LDMA) layers and PbS NPs/layered polymer composite materials}

P-LDMA layers were synthesized through irradiating the LDMA with a dose rate of $8.31 \mathrm{kGy} / \mathrm{h}$ in the field of a ${ }^{60} \mathrm{Co} \gamma$-ray source $\left(7 \times 10^{4}\right.$ curies) for $16 \mathrm{~h}$. Irradiation temperature was in the range $25-30{ }^{\circ} \mathrm{C}$. Subsequent to irradiation, the samples were put into an evacuated vial and an excess of $\mathrm{H}_{2} \mathrm{~S}$ gas was injected. The gas-solid reaction was carried out for $30 \mathrm{~min}$ at room temperature to enable $\mathrm{Pb}^{2+}$ ions to be 
converted into PbS NPs.

\subsection{Characterization}

TEM images and SAED patterns were obtained by employing a JEOL-2010 transmission electron microscope, using an accelerating voltage of 200 $\mathrm{kV} .{ }^{13} \mathrm{C}$ nuclear magnetic resonance (NMR) spectra were recorded on a Varian solid state NMR Infinity Plus 400 instrument. Powder X-ray diffraction (XRD) data were collected on a Rigaku D/Max-2500 X-ray diffractometer using a $\mathrm{Cu}$ target radiation source. Fourier transform infrared (FTIR) spectra were taken on Nicolet Avatar 360 FTIR infrared spectrophotometer. Fluorescence experiments were performed on a PTI Fluorescence Master System. X-ray crystallography: Diffraction data were collected on a Rigaku R-AXIS RAPID diffractometer (Mo K $\alpha$ radiation, graphite monochromator) in the $\omega$ rotation scan mode. The structure determination was done by direct methods by using SHELXL 5.01v and refined with full-matrix least squares on $F^{2}$. A summary of the crystallographic data and structure refinement is provided in the Electronic Supplementary Material (ESM), Table S-1.

\section{Results and discussion}

The preparation procedure of the PbS NPs/layered polymer composites is illustrated schematically in Fig. 1. Firstly, LDMA monomers, which acted as the precursor for the composite layers, were synthesized by dissolution of lead oxide in hot aqueous solution of MA. After adding ethanol to the aqueous solution, the LDMA molecules slowly self-assembled into layered structures. The resulting LDMA layers were formulated as $\left[\mathrm{Pb}\left(\mathrm{C}_{4} \mathrm{H}_{5} \mathrm{O}_{2}\right)_{2} \cdot \mathrm{H}_{2} \mathrm{O}\right]$ based on single crystal X-ray diffraction analysis. Secondly, the LDMA layers were polymerized to P-LDMA layers by ${ }^{60} \mathrm{Co} \quad \gamma$-ray irradiation. Since there are two $\mathrm{C}=\mathrm{C}$ bonds in each LDMA molecule, a crosslinked structure is produced in the polymerization procedure, which enhances the mechanical strength and the thermal and chemical stability of the polymer layers. Finally, PbS NPs were generated in situ by exposing the P-LDMA layers to $\mathrm{H}_{2} \mathrm{~S}$ gas at room temperature. The layered P-LDMA host effectively

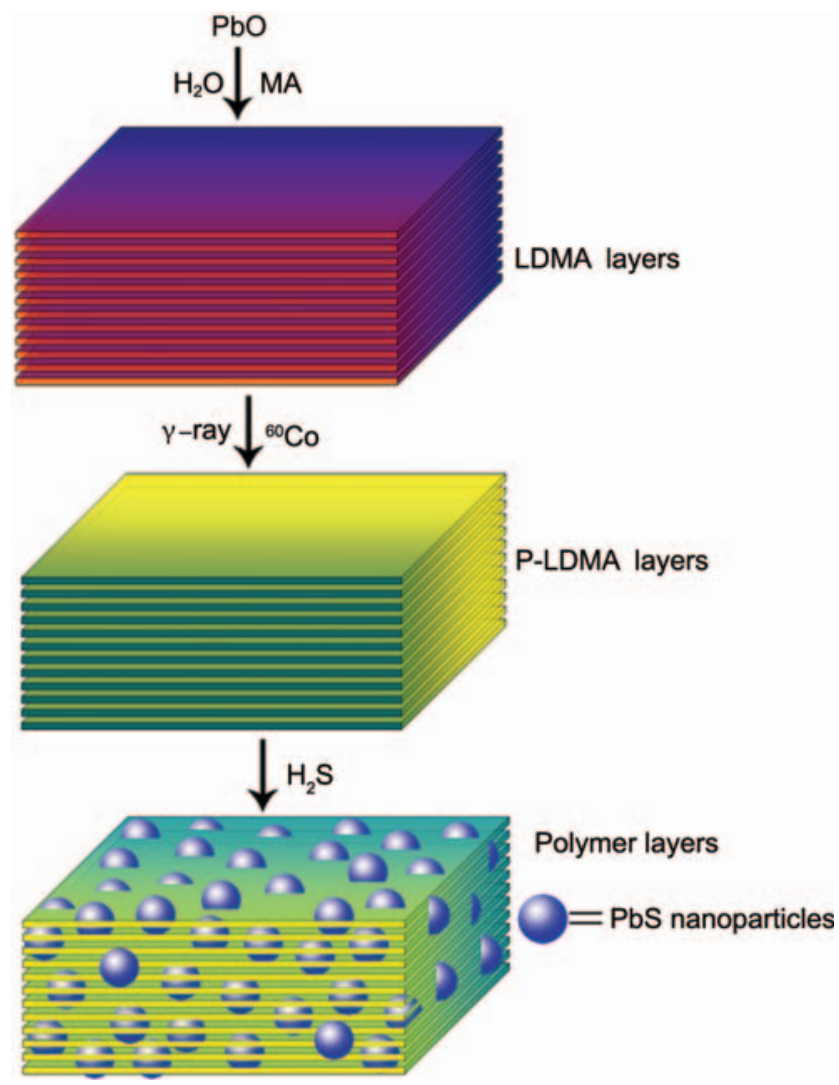

Figure 1 Synthetic pathway for the preparation of PbS NPs/layered polymer composite materials

prevents NPs from growing further after nucleation, and therefore uniform and well-dispersed PbS NPs were obtained.

The layered structure of the LDMA sample was confirmed by powder XRD measurements. Figure 2(a) shows the XRD pattern of LDMA. The largest diffraction peak at $2 \theta=7.15^{\circ}$, and successive periodic diffraction peaks assignable to those from the (100) to (700) planes, demonstrated a $d$-spacing of 1.23 $\mathrm{nm}$ (based on the Bragg equation $(\lambda=2 d \sin \theta$, $\lambda=0.15406)$ ). Single crystal XRD analysis was performed on LDMA in order to obtain the detailed molecular structure (Fig. 3). LDMA adopts a 2-D infinite structure wherein lead complex layers are arranged parallel to the $b c$ plane. Adjacent lead atoms are linked via methacrylate units. The sheets are stacked in the crystallographic $a$ direction, with no noticeable inter-sheet interactions. The layer distance is about $1.2 \mathrm{~nm}$, which is consistent with the conclusion drawn from the powder XRD pattern. The laminated structure suggests that crystal growth 
perpendicular to the sheet plane is slower than that in the plane, because of the weaker interactions in that direction [30].

Since the LDMA layers are continuous in two dimensions and the ligand (MA) used in our experiments is easily polymerized, the layered coordination networks can be utilized for the construction of layered polymer materials. In order to convert LDMA layers into P-LDMA layers without altering their layered structure, we employed a $y$ ray irradiation method to polymerize LDMA. Figure 2(b) shows the XRD pattern of the sample after $y$-irradiation, indicating the sample maintains a layered structure after polymerization. No reduction in basal spacing is observed, suggesting that the polymerization only occurred within every layer instead of between layers.

In order to confirm the successful preparation of P-LDMA from LDMA, ${ }^{13} \mathrm{C}$ solid-state NMR spectroscopy (Fig. 4(a)) was used to determine the structural changes in the sample after irradiation. Three features are observed that provide evidence of $\gamma$-irradiated polymerization within the layered materials. (1) The intensities of resonances at 175 and $143 \mathrm{ppm}$ assigned to $\mathrm{sp}^{2}$ hybridized carbon atoms of $\mathrm{C}\left(\mathrm{CH}_{3}\right)=\mathrm{CH}_{2}$ decrease significantly after $\gamma$-ray exposure, indicating $\mathrm{C}=\mathrm{C} \rightarrow \mathrm{C}-\mathrm{C}$ conversion; (2) Compared with the ${ }^{13} \mathrm{C}$ NMR spectrum of LDMA layers, it is found that a new peak at 49

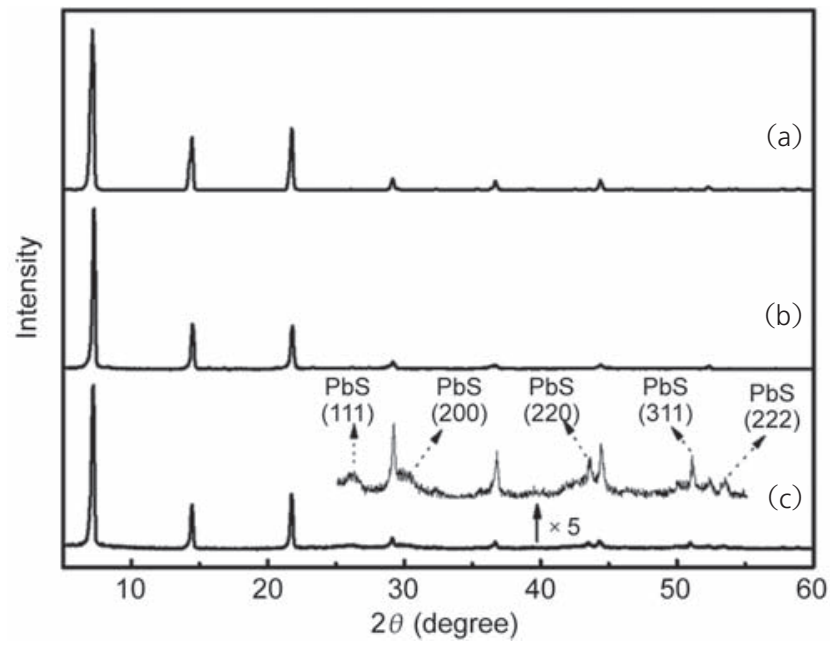

Figure 2 Powder XRD patterns of LDMA layers (a), P-LDMA layers (b) and PbS NPs/layered polymer composite materials (c). (In order to see clearly, the peaks between $25^{\circ}$ to $55^{\circ}$ in Fig. 2(c) are enlarged 5 times) ppm emerges in the spectrum of the sample after $\gamma$-irradiation. This extra peak can be attributed to the new $\mathrm{sp}^{3}$ hybridized carbon atoms that are formed by reaction of the $\mathrm{sp}^{2}$ hybridized carbon atoms in the $y$ irradiation process; (3) The $\mathrm{C}=\mathrm{O}$ resonance is shifted from 177 to $186 \mathrm{ppm}$ after $\gamma$-irradiation, consistent with methacrylate polymerization [31]. It should also be noted that the signals at 175 and 143 ppm do not disappear completely after irradiation, illustrating that the polymerization reaction is incomplete. To

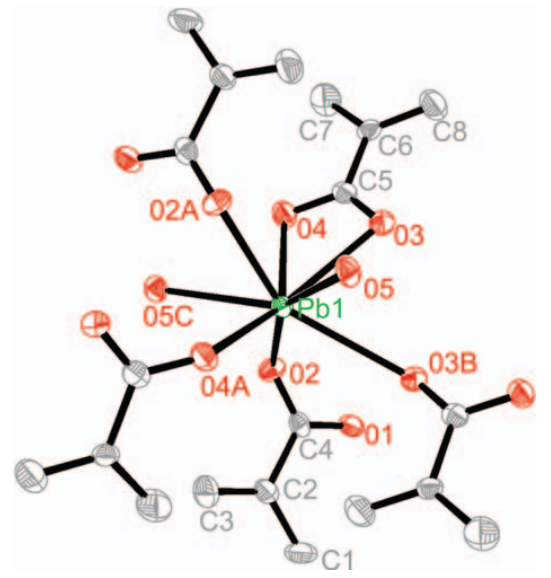

(a)

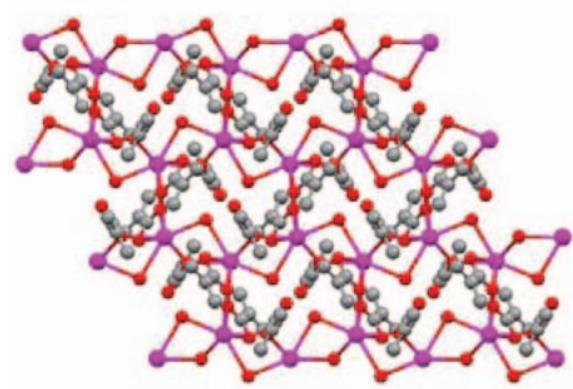

(b)
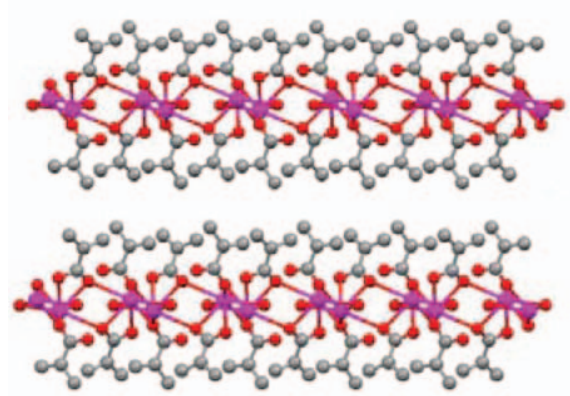

(c)

Figure 3 (a) ORTEP plot of the molecular structure of $\left[\mathrm{Pb}\left(\mathrm{C}_{4} \mathrm{H}_{5} \mathrm{O}_{2}\right)_{2} \cdot \mathrm{H}_{2} \mathrm{O}\right]$, emphasizing the coordination environment about lead ( $\mathrm{H}$ atom labels omitted for clarity); (b) view of one LDMA layer down the crystallographic a axis; (c) layered structure of LDMA, viewed along the $c$ axis (pink spheres, lead; red spheres, oxygen; gray spheres, carbon) 
perform quantitative analysis, FTIR spectra were recorded. As shown in Fig. 4(b), the $C=C$ stretch $\left(1643 \mathrm{~cm}^{-1}\right)$ obviously weakens in intensity after $y$-irradiation, suggesting that polymerization occurs. The carboxylate band at $1520 \mathrm{~cm}^{-1}$ was selected as the internal standard peak. By comparing the intensities of the $\mathrm{C}=\mathrm{C}$ bond peak before and after irradiation, we calculated that the degree of polymerization amounted to $78 \%$. Since there are two $\mathrm{C}=\mathrm{C}$ bonds in each LDMA molecule, Therefore, from the polymerization degree we can draw the conclusion that there was at least one double bond in each LDMA molecule participating in the polymerization. Moreover, for 56 percent of the LDMA molecules, the two double bonds were both polymerized, which suggests that cross-linked structures were produced in the polymerization procedure.

The stabilities of the samples are significantly improved after polymerization. For example, the starting LDMA precursors are soluble in hot water and numerous polar organic solvents, whereas the P-LDMA samples are quite inert in these solvents. Furthermore, LDMA samples melt at $74.6{ }^{\circ} \mathrm{C}$, whereas the P-LDMA display good thermal stability, with thermal decomposition at about $414{ }^{\circ} \mathrm{C}$. The very different properties of LDMA and P-LDMA layers can be attributed to the successful polymerization on $\gamma$-irradiation and the cross-linked structures generated in this process. Such layered polymer materials cannot only act as alternatives to layered inorganic materials in some caustic environments, but also should allow the generation of NIR luminescent $\mathrm{PbS}$ nanoparticels without aggregation in the polymer matrix. That is because the $\mathrm{Pb}^{2+}$ ions, which are introduced before polymerization, are dispersed uniformly in the polymer layers and the layered structures can effectively prevent the $\mathrm{PbS}$ NPs from growing further after nucleation.

The $\mathrm{Pb}^{2+}$ ions act as centers in the polymer chains for the introduction of dispersed functional PbS NPs into the polymer layers. PbS NPs were prepared in situ by exposing the polymer layers to $\mathrm{H}_{2} \mathrm{~S}$ gas at room temperature. The samples rapidly turned from white to black. In the FTIR spectra (Fig. 4(b)) of the samples after sulfuration, the presence of the characteristic bands at around $1700 \mathrm{~cm}^{-1}$ attributed to the protonated carboxylic acid groups indicates the successful formation of PbS NPs. The morphology of the PbS NPs was examined by transmission electron microscopy (TEM). As shown in Fig. 5, the dense PbS NPs with average diameter of approximately $6 \mathrm{~nm}$ are separated from each other and well dispersed in the polymer lamellae (the size of the nanoparticles is somewhat bigger than that in our previous work using P-LDMA nanofibers [17], which can be mainly attributed to the less compact nature of the P-LDMA layers compared with the LDMA nanofibers). One of the numerous key challenges in producing nanocomposites that exhibit the desired behavior is be able to control the dispersion of NPs in polymeric

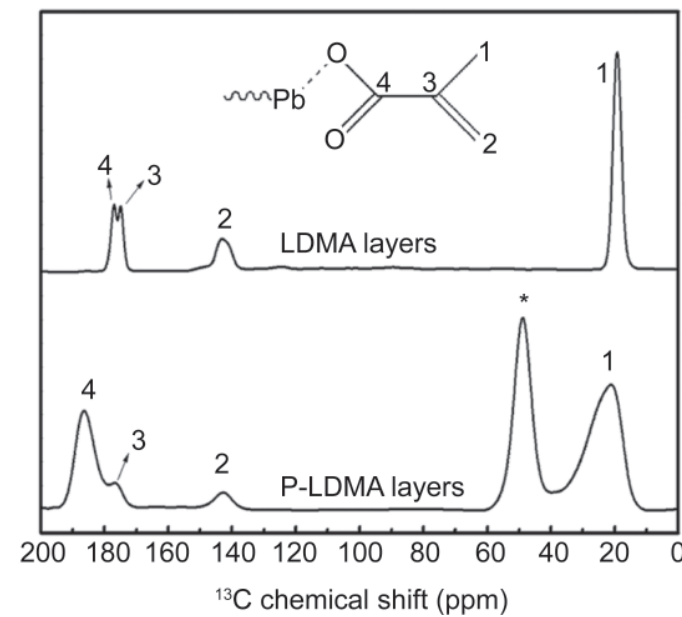

(a)

Figure 4 (a) ${ }^{13} \mathrm{C}$ NMR spectra of LDMA layers and P-LDMA layers; (b) FTIR spectra of LDMA layers, P-LDMA layers and PbS NPs/layered polymer composite materials

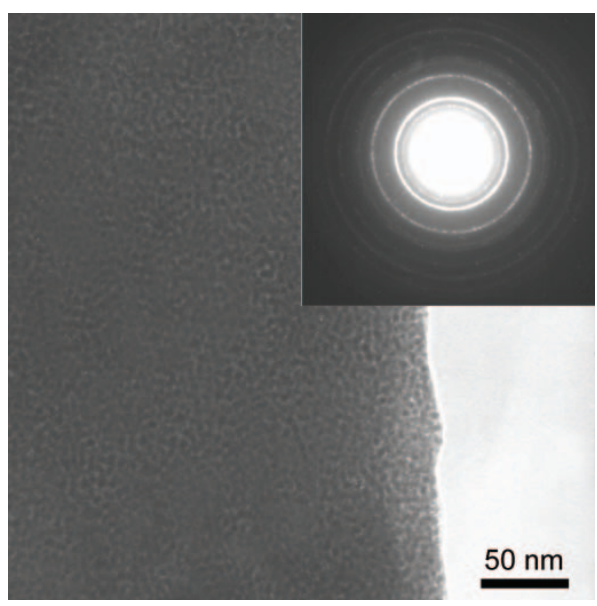

Figure 5 TEM image of PbS NPs/layered polymer composite materials (viewed along the a axis). Inset: SAED pattern of the PbS NPs 
hosts. In our experiments, the PbS particles generated are small in size and well-dispersed in the polymer layers; this can be attributed to three major reasons. Firstly, since these layered polymer materials are prepared from metal-organic coordination networks, the $\mathrm{Pb}^{2+}$ ions are dispersed quite uniformly in the polymer layers. Secondly, both the relatively low degree of compactness of the layered polymer and the vacuum treatment before sulfuration (see paragraph 1.3 in the "Experimental" section), facilitate the diffusion of gaseous $\mathrm{H}_{2} \mathrm{~S}$ into the polymer matrix. Therefore, relatively uniform $\mathrm{PbS}$ NPs can be obtained. Thirdly, after the reaction with $\mathrm{H}_{2} \mathrm{~S}$, some carboxyl groups of the polymer layers are still bonded to the $\mathrm{Pb}^{2+}$ ions on the particle surface (in the FTIR spectrum of the PbS NPs/layered polymer materials, the band attributed to carboxylate at 1520 $\mathrm{cm}^{-1}$ is still present after sulfuration), the crosslinked polymer layers networks can prevent the $\mathrm{PbS}$ particles from growing further after nucleation, resulting in their small size. Data obtained from the selected-area electron diffraction (SAED) pattern (inset of Fig. 5) show that the PbS NPs have a cubic fcc rock-salt structure. Detailed structural analysis of the PbS NPs in the polymer layers was further carried out by powder XRD. From Fig. 2(c), we can see that, in addition to the strong periodic peaks which confirm the layered structure still remains, some other weaker peaks also appeared at $2 \theta: 25.86^{\circ}$, $29.98^{\circ}, 43.44^{\circ}, 50.96^{\circ}$, and $53.36^{\circ}$, which can be indexed to the cubic fcc rock-salt structure of a $\mathrm{PbS}$ phase with the cell parameter $a=5.936 \AA$ for PbS NPs (JCPDS 05-0592).

Figure 6 shows the room temperature photoluminescence spectrum of the PbS NPs/layered polymer composite materials in the NIR $\left(\lambda_{e x}=550\right.$ $\mathrm{nm}$ ) region. The peak maximum of the NIR emission was found at about $1355 \mathrm{~nm}$. We believe that this peak is due to band-edge emission and the emission maximum is thus size-tunable. The band gap value $(\Delta E)$ of small sized particles is normally calculated from the hyperbolic band model using the equation [32] ,

$$
\Delta E=\left[E_{\mathrm{g}}{ }^{2}+2 \hbar^{2} E_{\mathrm{g}}(\pi / R)^{2} / m^{*}\right]^{1 / 2}
$$

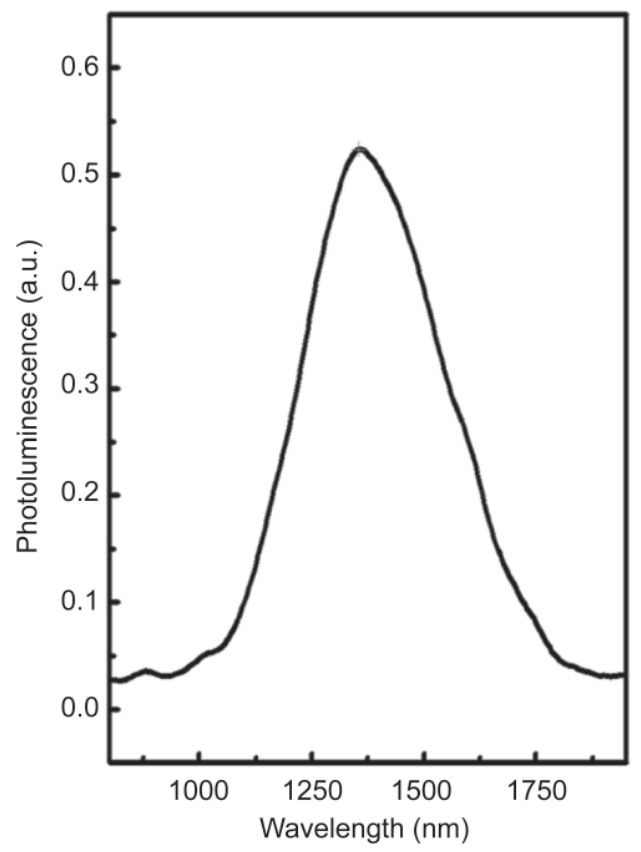

Figure 6 PL spectrum of PbS NPs/layered polymer composite materials

where $E_{\mathrm{g}}$ is the band gap of the bulk PbS $(0.41 \mathrm{eV})$, $m^{*} / m_{\mathrm{e}}=0.085\left(m^{*}\right.$ is the actual electron mass), and $R$ is the particle radius. We have thus calculated the expected $\Delta E$ value using the average particle size obtained from TEM analysis. The calculated band gap value is $0.98 \mathrm{eV}$ (hence according to $E=h c / \lambda$, $\lambda=1265 \mathrm{~nm}$ ), which is in good agreement with our observed NIR emission maximum. This confirms the quantum size effect and strong confinement compared with bulk PbS ( 3000 nm). The NIR luminescent property of the PbS NPs further confirms that the cross-linked P-LDMA layers and the bonds between the particle surface and the carboxyl groups not only enhance the mechanical strength and thermal and chemical stability of the composite materials but also favor formation of relatively uniform PbS NPs with low density of surface states throughout the entire polymer matrix. These results point to the possibility of using this composite system to create light-emitting structures for optical elements at telecommunications wavelengths from 1300 to $1550 \mathrm{~nm}$. However, it should be pointed out that the photoluminescence peak of PbS NPs observed in this work is relatively broad compared with semiconductor NPs prepared by organometallic approaches [7, 33-35], indicating such in situ methods should be further improved in order to 
achieve semiconductor nanoparticles with a narrow size distribution.

\section{Conclusions}

We have demonstrated the preparation of 2-D metalorganic coordination networks and their conversion into PbS NPs/layered polymer composite materials through the combined use of $\gamma$-ray irradiation and a gas/solid reaction. In this approach, $\gamma$-irradiation offers an ideal means to fabricate polymer materials from coordination networks without introducing any other reagent. Such PbS NPs/layered polymer composite materials should have potential applications in various fields, as they combine the advantages of both layered structures and PbS NPs/ polymer hybrid materials. Recently, we successfully synthesized one-dimensional (1-D) NPs/polymer composite nanofibers by $\gamma$-irradiation and a gas/solid reaction. In the present paper, this methodology is extended to 2-D layered materials. We anticipate that the method is universal and can be adapted for the conversion of different coordination networks with double bonds into diverse polymer or multifunctional NPs/polymer nanostructures, which would have potential applications in fabricating devices with optical, electrical, and magnetic properties.

\section{Acknowledgments}

This work has been supported by the National Natural Science Foundation of China (Nos. 20504011, 20534040, 50703046, and 20674026), and the National Basic Research Program of China (2007CB936402).

Electronic Supplementary Material: Supplementary material is available in the online version of this article at http://dx.doi.org/10.1007/s12274-008-8019-2 and is accessible free of charge.

\section{References}

[1] Balazs, A. C.; Emrick, T.; Russell, T. P. Nanoparticle polymer composites: Where two small worlds meet. Science 2006, 314, 1107-1110.

[2] Wise, F. W. Lead salt quantum dots: The limit of strong quantum confinement. Acc. Chem. Res. 2000, 33, 773780.

[3] Peterson, J. J.; Krauss, T. D. Fluorescence spectroscopy of single lead sulfide quantum dots. Nano Lett. 2006, 6, 510-514.

[4] Patel, A. A.; Wu, F.; Zhang, J. Z.; Torres-Martinez, C. L.; Mehra, R. K.; Yang, Y.; Risbud, S. H. Synthesis, optical spectroscopy and ultrafast electron dynamics of PbS nanoparticles with different surface capping. J. Phys. Chem. B 2000, 104, 11598-11605.

[5] Wang, Y. Nonlinear optical properties of nanometer-sized semiconductor clusters. Acc. Chem. Res. 1991, 24, 133139.

[6] Liu, B.; Li, H.; Chew, C. H.; Que, W.; Lam, Y. L.; Kam, C. H.; Gan, L. M.; Xu, G. Q. PbS-polymer nanocomposite with third-order nonlinear optical response in femtosecond regime. Mater. Lett. 2001, 51, 461-469.

[7] Hines, M. A.; Scholes, G. D. Colloidal PbS nanocrystals with size-tunable near-infrared emission: Observation of post-synthesis self-narrowing of the particle size distribution. Adv. Mater. 2003, 15, 1844-1849.

[8] Lim, W. P.; Low, H. Y.; Chin, W. S. IR-luminescent PbSpolystyrene nanocomposites prepared from random ionomers in solution. J. Phys. Chem. B 2004, 108, 1309313099.

[9] Bakueva, L.; Musikhin, S.; Hines, M. A.; Chang, T. W. F.; Tzolov, M.; Scholes, G. D.; Sargent, E. H. Size-tunable infrared (1000-1600 nm) electroluminescence from PbS quantum-dot nanocrystals in semiconducting polymer. Appl. Phys. Lett. 2003, 82, 2895-2897.

[10] Winiarz, J. G.; Zhang, L.; Park, J.; Prasad, P. N. Inorganic: Organic hybrid nanocomposites for photorefractivity at communication wavelengths. J. Phys. Chem. B 2002, 106, 967-970.

[11] Bakueva, L.; Gorelikov, I.; Musikhin, S.; Zhao, X. S.; Sargent, E. H.; Kumacheva, E. PbS quantum dots with stable efficient luminescence in the near-IR spectral range. Adv. Mater. 2004, 16, 926-929.

[12] McDonald, S. A.; Konstantatos, G.; Zhang, S.; Cyr, P. W.; Klem, E. J. D.; Levina, L.; Sargent, E. H. Solutionprocessed $\mathrm{PbS}$ quantum dot infrared photodetectors and photovoltaics. Nat. Mater. 2005, 4, 138-142.

[13] Ellingson, R. J.; Beard, M. C.; Johnson, J. C.; Yu, P.; Micic, O. I.; Nozik, A. J.; Shabaev, A.; Efros, A. L. Highly efficient multiple exciton generation in colloidal PbSe and PbS quantum dots. Nano Lett. 2005, 5, 865-871.

\section{国些 Springer}


[14] Hoogland, S.; Sukhovatkin, V.; Howard, I.; Cauchi, S.; Levina, L.; Sargent, E. H. A solution-processed $1.53 \mu \mathrm{m}$ quantum dot laser with temperature-invariant emission wavelength. Opt. Express 2006, 14, 3273-3281.

[15] Lü, C.; Guan, C.; Liu, Y.; Cheng, Y.; Yang, B. PbS/polymer nanocomposite optical materials with high refractive index. Chem. Mater. 2005, 17, 2448-2454.

[16] Wang, S.; Yang, S. Preparation and characterization of oriented PbS crystalline nanorods in polymer films. Langmuir 2000, 16, 389-397.

[17] Cui, T.; Cui. F.; Zhang, J.; Wang, J.; Huang, J.; Lü, C.; Chen, Z.; Yang, B. From monomeric nanofibers to PbS/ polymer composite nanofibers through the combined use of $\gamma$-irradiation and gas/solid reaction. J. Am. Chem. Soc. 2006, 128, 6298-6299.

[18] Auerbach, S. M.; Carrado, K. A.; Dutta, P. K., Eds. Handbook of Layered Materials; Marcel Dekker: New York, 2004.

[19] Su, W.; Hong, M.; Weng, J.; Cao, R.; Lu, S. A semiconducting lamella polymer $\left[\left\{\mathrm{Ag}\left(\mathrm{C}_{5} \mathrm{H}_{4} \mathrm{NS}\right)\right\}_{n}\right]$ with a graphite-like array of silver (I) lons and its analogue with a layered structure. Angew. Chem. Int. Ed. 2000, 39, 2911-2914.

[20] Darder, M.; Aranda, P.; Ruiz-Hitzky, E. Bionanocomposites: A new concept of ecological, bioinspired, and functuional hybrid materials. Adv. Mater. 2007, 19, 1309-1319.

[21] Clearfield, A. Role of ion exchange in solid-state chemistry. Chem. Rev. 1988, 88, 125-148.

[22] Kumar, C. V.; Chaudhari, A. Proteins immobilized at the galleries of layered $\alpha$-zirconium phosphate: Structure and activity studies. J. Am. Chem. Soc. 2000, 122, 830837.

[23] Bonhomme, F.; Alam, T. M.; Celestian, A. J.; Tallant, D. R.; Boyle, T. J.; Cherry, B. R.; Tissot, R. G.; Rodriguez, M. A.; Parise, J. B.; Nyman, M. Tribasic lead maleate and lead maleate: Synthesis and structural and spectroscopic characterizations. Inorg. Chem. 2005, 44, 7394-7402.

[24] Pan, L.; Huang, X.; Li, J.; Wu, Y.; Zheng, N. Novel singleand double-layer and three-dimensional structures of rare-earth metal coordination polymers: The effect of lanthanide contraction and acidity control in crystal structure formation. Angew. Chem. Int. Ed. 2000, 39, 527-530.
[25] Xu, H.; Li, Y. The organic ligands as template: The synthesis, structures and properties of a series of the layered structure rare-earth coordination polymers. J. Mol. Struct. 2004, 690, 137-143.

[26] Rogow, D. L.; Zapeda, G.; Swanson, C. H.; Fan, X.; Campana, C. F.; Oliver, A. G.; Oliver, S. R. J. A metalorganic framework containing cationic inorganic layers: $\mathrm{Pb}_{2} \mathrm{~F}_{2}\left[\mathrm{C}_{2} \mathrm{H}_{4}\left(\mathrm{SO}_{3}\right)_{2}\right]$. Chem. Mater. 2007, 19, 4658-4662.

[27] Noro, S. I.; Horike, S.; Tanaka, D.; Kitagawa, S.; Akutagawa, T.; Nakamura, T. Flexible and shape-selective guest binding at $\mathrm{Cu}^{\prime \prime}$ axial sites in 1-dimensional $\mathrm{Cu}^{\prime \prime}-1,2-$ bis(4-pyridyl)ethane coordination polymers. Inorg. Chem. 2006, 45, 9290-9300.

[28] Shi, F. N.; Cunha-Silva, L.; Sá Ferreira, R. A.; Mafra, L.; Trindade, T.; Carlos, L. D.; Almeida Paz, F. A.; Rocha, J. Interconvertable modular framework and layered lanthanide (III)-etidronic acid coordination polymers. J. Am. Chem. Soc. 2008, 130, 150-167.

[29] Zhao, Y.; Hong, M.; Liang, Y.; Cao, R.; Li, W.; Weng, J.; Lu, S. A paramagnetic lamellar polymer with a high semiconductivity. Chem. Commun. 2001, 1020-1021.

[30] Williams, D. J.; Maginn, S. J.; Davey, R. J. The X-ray crystal structure of lead acetophthalate, $\mathrm{Pb}\left(\mathrm{CH}_{3} \mathrm{COO}\right)_{2}$. $4\left[\mathrm{PbC}_{6} \mathrm{H}_{4}(\mathrm{COO})_{2}\right]$. Polyhedron 1994, 13, 1683-1688.

[31] Sellinger, A.; Weiss, P. M.; Nguyen, A.; Lu, Y.; Assink, R. A.; Gong, W.; Brinker, C. J. Continuous self-assembly of organic-inorganic nanocomposite coatings that mimic nacre. Nature 1998, 394, 256-260.

[32] Wang, Y.; Suna, A.; Mahler, W.; Kasowski, R. PbS in polymers. From molecules to bulk solids. J. Chem. Phys. 1987, 87, 7315-7322.

[33] Murray, C. B.; Norris, D. J.; Bawendi, M. G. Synthesis and characterization of nearly monodisperse $\mathrm{CdE}(\mathrm{E}=\mathrm{S}$, Se, Te) semiconductor nanocrystallites. J. Am. Chem. Soc. 1993, 115, 8706-8715.

[34] Peng, X.; Wickham, J.; Alivisatos, A. P. Kinetics of II-VI and III-V colloidal semiconductor nanocrystal growth: "Focusing" of size distributions. J. Am. Chem. Soc. 1998, 120, 5343-5344.

[35] Peng, X.; Manna, L.; Yang, W.; Wickham, J.; Scher, E.; Kadavanich, A.; Alivisatos, A. P. Shape control of CdSe nanocrystals. Nature 2000, 404, 59-61. 